\title{
The Impact of Entrepreneurial Risk Aversion on Wages in General Equilibrium
}

\author{
Ying Feng \\ James E. Rauch
}

CESIFO WORKING PAPER NO. 5358

CATEGORY 11: INDUSTRIAL ORGANISATION

MAY 2015

An electronic version of the paper may be downloaded

- from the SSRN website:

- from the RePEc website:

- from the CESifo website:

www.SSRN.com

www.RePEc.org

www.CESifo-group.org/wp 


\title{
The Impact of Entrepreneurial Risk Aversion on Wages in General Equilibrium
}

\begin{abstract}
One of the leading theories of entrepreneurship is that less risk averse individuals become entrepreneurs and more risk averse individuals become their employees. Kihlstrom and Laffont (1979) formalized this insight in an elegant and widely taught general equilibrium model. However, their model has not been further developed. A reason may be that their main comparative static result, that an economy-wide increase in risk aversion lowers the equilibrium wage, appeared to require the assumption that all agents had identical risk aversion index, throwing out their motivating insight and indicating that the model is intractable. In this note we prove this comparative static result on risk aversion and wages in general equilibrium, retaining agent heterogeneity in risk aversion and the endogenous division of agents into less risk averse entrepreneurs and more risk averse workers, without adding any assumptions not already in the original paper. Besides the intrinsic value of the result, we hope to increase the usefulness of the Kihlstrom and Laffont (1979) model for other researchers and to facilitate improvement in its exposition for the many graduate courses in which it is taught.
\end{abstract}

JEL-Code: L260.

Keywords: entrepreneurship, risk aversion.

Ying Feng

Department of Economics

University of California, San Diego

9500 Gilman Drive

USA - 92093-0508 La Jolla CA

jenyfeng@ucsd.edu
James E. Rauch

Department of Economics

University of California, San Diego

9500 Gilman Drive

USA - 92093-0508 La Jolla CA

jrauch@ucsd.edu

(econweb.ucsd.edu/jrauch/)

May 17, 2015

Our thanks to Alexis Akira Toda and Richard Kihlstrom for helpful comments. We are responsible for any errors. 


\section{Introduction}

Kihlstrom and Laffont (1979) (hereafter KL) and Lucas (1978) developed the two most popular theories of entrepreneurship (Parker 2009, chapter 2). According to KL, entrepreneurship is determined by risk aversion, with the less risk averse agents employing the more risk averse agents. According to Lucas (1978), entrepreneurship is determined by managerial talent, with the more talented agents employing the less talented agents. Written at the same time, KL and Lucas (1978) have now accumulated 1,553 and 2,627 Google Scholar citations, respectively (accessed March 17, 2015). However, the otherwise parallel paths of these papers have diverged in one important respect: whereas both papers have inspired vast amounts of empirical work, only Lucas (1978) has been extended theoretically. ${ }^{1}$ To take a couple of widely cited examples, Evans and Jovanovic (1989) extended Lucas's model to the case of finance constraints, and Maksimovic and Phillips (2002) extended it to two sectors across which managerial talent differs.

There could, of course, be many reasons why subsequent authors have found Lucas (1978) more amenable to theoretical development than KL. Here we focus on one possible reason: readers were discouraged by the apparent intractability of the KL model for comparative static analysis. Their only comparative static result is that an increase in risk aversion lowers the equilibrium wage. ${ }^{2}$ This result is intuitive: greater risk aversion causes agents to prefer wage work over entrepreneurship, and also causes entrepreneurs to hire fewer workers. Nevertheless, in order to prove this result KL set aside the motivating insight of their model that less risk averse agents employ more risk averse agents. Instead, they in effect assumed that all agents had identical risk aversion index.

In this note we will prove the comparative static result on risk aversion and wages in general equilibrium, retaining agent heterogeneity in risk aversion and the endogenous division of agents into less risk averse entrepreneurs and more risk averse workers, without adding any assumptions not already in KL. Besides the intrinsic value of the result, we hope to increase the usefulness of KL for other researchers and to facilitate improvement in its exposition for the many graduate courses

\footnotetext{
${ }^{1}$ Our assertion that KL has not been extended theoretically is based on our review of papers that cite it that themselves have at least 25 Google Scholar citations. This is not to say that all such papers are purely empirical, just that those with theoretical models invoke KL rather than build on their model.

${ }^{2}$ They also include in their section on comparative statics the result that firm employment decreases with the risk aversion of the entrepreneur (theorem 3), but they attribute this result to Baron (1970).
} 
in which it is taught.

In the next section we will present the KL model briefly. In section 3 we will prove our result, which is a revised version of KL theorem 4. In the following section we will show by example that an interior solution to the KL model exists. Section 5 concludes.

\section{KL Model Setup}

KL identify the set of agents with the interval $[0,1]$. Individual $\alpha \in[0,1]$ has the von Neumann Morgenstern utility function $u(I, \alpha)$ where $I \in[0, \infty)$ represents income. Agents are characterized by the Arrow-Pratt absolute risk aversion measure $r(I, \alpha)=-\frac{u_{I I}(I, \alpha)}{u_{I}(I, \alpha)}$, where $r(I, \alpha)$ is a strictly increasing function of $\alpha$ for each $I .^{3}$

Each agent can become an entrepreneur and use without cost a technology defined by a continuous production function $y=g(L, x)$ where $y \geq 0$ is output, $L \geq 0$ is the labor input and $x$ is the value taken by a nondegenerate random parameter $\tilde{x}$ with support $[0, \bar{x}], 0<\bar{x}<+\infty$. The objective stochastic distribution of $\tilde{x}$ is assumed to be the same for all firms. KL also assume the marginal product $g_{L}$ is continuous and positive, and the second derivative $g_{L L}$ is continuous and nonpositive, on $[0,+\infty) \times[0, \bar{x}]$. Thus $g$ exhibits nonincreasing returns to scale for each $x$. In addition, $g(0, x)=g(L, 0)=0$ for all $x \in[0, \bar{x}]$ and $L \in[0,+\infty)$, while $g(L, x)>0$ on $(0,+\infty) \times(0, \bar{x}]$.

The price of output is 1 and labor is hired at a competitive wage $w$. Agents can become entrepreneurs and receive an uncertain income of $g(L, \tilde{x})-w L$, or they can become workers and receive the market wage $w$. To avoid the problem of bankruptcy, KL assume that all individuals begin with $A$ units of income and that entrepreneurs are unable to hire workers who cannot be paid if $\tilde{x}=0$. Thus $L \leq \frac{A}{w}$ must hold.

The equilibrium in KL is then pinned down by three key steps. First, an individual who becomes

\footnotetext{
${ }^{3} \mathrm{KL}$ add the assumption that $r$ is strictly increasing rather than non-decreasing in $\alpha$ in a Lemma (p. 725) that precedes their proof of existence of equilibrium (theorem 1).
} 
an entrepreneur chooses employment $L(w, \alpha) \in[0, A / \omega]$ which solves ${ }^{4}$

$$
\operatorname{Max}_{L} E u(A+g(L, \tilde{x})-w L, \alpha)
$$

The assumptions on $u$ and $g$ guarantee that $L(w, \alpha)$ exists. If either $u_{I I}<0$ or $g_{L L}<0$, then $L(w, \alpha)$ will be unique. ${ }^{5}$

Second, the certainty equivalent wage $w(\alpha)$ of agent $\alpha$ is defined by ${ }^{6}$

$$
E u[A+g(L(w(\alpha), \alpha), \tilde{x})-w(\alpha) L(w(\alpha), \alpha), \alpha]=u(A+w(\alpha), \alpha)
$$

Thus, under market wage $w(\alpha)$, agent $\alpha$ is indifferent between work and entrepreneurship. Moreover, $w(\alpha)$ is continuous and monotonically decreasing: ${ }^{7}$ the more risk averse is the agent, the lower is the wage that makes him indifferent between entrepreneurship and wage work.

Finally, a general equilibrium where the labor market clears is characterized by a marginal entrepreneur $\hat{\alpha}$ such that ${ }^{8}$

$$
\int_{0}^{\hat{\alpha}} L(w(\hat{\alpha}), \alpha) \mathrm{d} \alpha=1-\hat{\alpha}
$$

where agents with $\alpha \in[0, \hat{\alpha}]$ are entrepreneurs and agents with $\alpha \in(\hat{\alpha}, 1]$ are workers.

Thus, knowing $\hat{\alpha}$, we know the equilibrium wage $w(\hat{\alpha})$, and knowing $w(\hat{\alpha})$, we know $L(w(\hat{\alpha}), \alpha)$ for every entrepreneur $\alpha \in[0, \hat{\alpha}]$. KL go on to demonstrate the uniqueness of this equilibrium in their theorem 2 .

\footnotetext{
${ }^{4}$ This is equation (3) on page 724 of KL.

${ }^{5}$ The existence of $L(w, \alpha)$ is guaranteed by the continuity of the expectation operation, $u$, and $g$, plus the fact that $L$ is bounded in $\left[0, \frac{A}{w}\right]$. If either $u_{I I}<0$ or $g_{L L}<0$, the objective function is strictly concave, so $L(w, \alpha)$ will be unique. This last assumption is made in both theorem 1 and theorem 2 of KL.

${ }^{6}$ This is equation (7) on page 724 of KL.

${ }^{7}$ Prior to theorem 1 proving existence of equilibrium, KL add the assumption that $u(I, \alpha)$ is everywhere a continuous function of $\alpha$, from which the continuity of $w(\alpha)$ follows.

${ }^{8}$ This is equation (14) on page 728 of KL. They use notation $\hat{\alpha}^{*}$ but we simplify it to $\hat{\alpha}$ for further use in our proof.
} 


\section{Risk Aversion and the Equilibrium Wage}

KL state their comparative static result as theorem 4, which we reproduce exactly here, noting that $\hat{\alpha}$ is defined by KL as the marginal entrepreneur:

Theorem 4: If (i) in equilibrium, all entrepreneurs are identical, (ii) either $g_{L L}<0$ or $u_{I I}<0$, (iii) $g(L, x)$ and $g_{L}(L, x)$ are both monotonically increasing (or decreasing) functions of $x$, and (iv) $L(w, \hat{\alpha})$ is an interior solution and a decreasing function of $w$, then an increase in the Arrow-Pratt absolute risk aversion measure $r(I, \hat{\alpha})$ for all $I$, lowers the equilibrium wage.

Unlike assumptions ii - iv, assumption i does not appear earlier in KL. To appreciate the importance of assumption i, recall that the division of agents between entrepreneurs and workers is endogenous. The only way, then, to guarantee that all entrepreneurs are identical in equilibrium is to assume that all agents are identical. In this case, equilibrium is only possible when all agents are indifferent between becoming entrepreneurs or becoming workers: otherwise there is either no demand for labor or no supply of labor. With the wage determining the labor demand of every identical firm, indifferent agents must divide between entrepreneurs and workers so as to provide enough firms to exactly employ all the agents who do not operate firms. That is, in equilibrium the wage adjusts so as to allow the division of indifferent agents between entrepreneurs and workers to clear the labor market.

As theorem 4 is written, it is clear that equation (3) no longer applies because $\hat{\alpha}$ no longer divides agents between entrepreneurs in $[0, \hat{\alpha}]$ and workers in $(\hat{\alpha}, 1]$. In other words, it is no longer the case that in equilibrium the less risk averse agents employ the more risk averse agents. Accordingly, KL do not use equation (3) in their proof of theorem 4. Moreover, their statement of theorem 4 is at odds with their remark 2 that immediately follows their statement of the theorem:

Remark 2: The intuitive basis for the result is as follows. Since, in equilibrium, workers are the most risk averse individuals, an economy-wide increase in risk aversion increases the supply of workers and this tends to lower the wage. This tendency is reinforced by demand changes implied by theorem 3 which applies because of assumption iii. 
Specifically, theorem 3 implies that an increase in the entrepreneurs' aversion to risk reduces the demand for labor.

Given assumption i, it is not meaningful to state that "in equilibrium, workers are the most risk averse individuals," and the economy-wide increase in risk aversion increases the supply of workers only in the sense that each (identical) firm employs fewer workers at a given wage. Our main goal in this note is thus to restate and prove theorem 4 so as to maintain the applicability of equation (3) and fulfill the original intent of KL as embodied in their remark 2 quoted above.

Let the assumptions in KL theorem 1 (existence), KL theorem 2 (uniqueness), and KL theorem $3\left(L(w, \alpha)\right.$ decreasing in entrepreneurial risk aversion) hold $^{9}$, as well as the assumption in their Lemma (risk aversion increasing in $\alpha$, not merely non-decreasing). We do not make assumption i above. We can then state and prove the following revised version of KL theorem $4^{10}$ :

THEOREM 4 (REVISED). An increase in the Arrow-Pratt absolute risk aversion measure $r(I, \alpha)$ for all $\alpha \in[0,1]$ and for all $I$ lowers the equilibrium wage.

PROOF: Recall that the certainty equivalent wage $w(\alpha)$ is a continuous and monotonically decreasing function of $\alpha$. We can therefore invert this function to obtain the continuous and monotonically decreasing function $\alpha(w)$, which gives the risk aversion index of the agent who is indifferent between wage work and entrepreneurship at wage $w$. We can then rewrite equation (3):

$$
\int_{0}^{\alpha(\hat{w})} L(\hat{w}, \alpha) \mathrm{d} \alpha=1-\alpha(\hat{w}),
$$

where $\hat{w}$ is the equilibrium wage. Now consider an economy-wide increase in risk aversion to $r^{\prime}(I, \alpha)>r(I, \alpha), \alpha \in[0,1]$. This implies $\alpha^{\prime}(\hat{w})<\alpha(\hat{w})$, because when risk aversion increases, holding $w$ constant, the risk aversion index of the marginal agent must decrease, since otherwise the marginal agent prefers wage work. $r^{\prime}(I, \alpha)>r(I, \alpha), \alpha \in[0,1]$ also implies $L^{\prime}(w, \alpha)<L(w, \alpha),{ }^{11}$

\footnotetext{
${ }^{9}$ Theorem 3 uses the assumption that $L(w, \alpha)<\frac{A}{w}$, but we actually do not need this assumption for our proof below.

${ }^{10}$ We interpret $\alpha$ as a pure index in our proof. An alternative proof, in which we interpret $\alpha$ as a parameter of the utility function, is available at http://acsweb.ucsd.edu/ yif014/proof2-note-kl1979.pdf. In the alternative proof we do not need assumption iii stated in KL theorem 4 above.

${ }^{11}$ If, for some $\alpha, L(w, \alpha)$ is not interior, then $L^{\prime}(w, \alpha)=L(w, \alpha)$.
} 
holding $w$ constant, since $L(w, \alpha)$ is decreasing in risk aversion by KL theorem 3 . Now rewrite equation (4) as equation (5):

$$
\int_{0}^{\alpha^{\prime}\left(\hat{w}^{\prime}\right)} L^{\prime}\left(\hat{w}^{\prime}, \alpha\right) \mathrm{d} \alpha=1-\alpha^{\prime}\left(\hat{w}^{\prime}\right),
$$

where $\hat{w}^{\prime}$ is the new equilibrium wage. Comparing the two equations, it is clear that at the equilibrium wage such that equation (4) holds $\left(\hat{w}^{\prime}=\hat{w}\right)$, the left-hand side of equation (5) is smaller than the right-hand side: there is an excess supply of labor. Because $\alpha^{\prime}(w)$ is decreasing and $L^{\prime}(w, \alpha)$ is decreasing in $w$, a fall in $\hat{w}^{\prime}\left(\hat{w}^{\prime}<\hat{w}\right)$ will restore labor market clearing. Q.E.D.

The seemingly innocuous assumption that $L(w, \alpha)$ is decreasing in $w$ conceals some subtlety. As KL explain in their remark 4 (p. 731), this holds if either (a) $r(I, \alpha)$ is a constant function of $I$, (b) $g(L, x)$ and $g_{L}(L, x)$ are both monotonically increasing (or decreasing) functions of $x$ and $r(I, \alpha)$ is a non-increasing function of $I$, or (c) $g(L, x)$ satisfies $g(L, x)=x h(L)$ and $-\frac{I u_{I I}(A+I, \alpha)}{u_{I}(A+I, \alpha)} \leq 1$ for all $I$. Those interested can read the proof of remark 4 in KL. Here, in order to build intuition, we simply note that these conditions are needed because changing $w$ also changes entrepreneurial income, which (in general) affects their risk aversion and therefore their labor demand. For example, consider assumption (b). If $w$ increases, entrepreneurial income decreases, hence risk aversion increases (or remains unchanged), reinforcing the negative effect on labor demand.

\section{An Example with an Interior Solution}

As mentioned above, in theorem $3 \mathrm{KL}$ demonstrate that $L(w, \alpha)$ is decreasing in entrepreneurial risk aversion, as indexed by $\alpha$. Naturally this result requires that $L(w, \alpha)$ is interior. Although KL are able to prove that $L(w, \alpha)>0$ in equilibrium, they have to assume $L(w, \alpha)<\frac{A}{w}$. To prove that the comparative static result in theorem 3 is not vacuous, we will now provide an example in which we prove that $L(w, \alpha)$ is interior in equilibrium. We hope this example will also be useful for pedagogy.

Let $u(I, \alpha)=I^{1-\alpha}$ where $I \in[0, \infty)$ and $\alpha \in(0,1)$. This functional form satisfies the assumptions 
made on $u$ and risk attitudes: if $\alpha>\beta$, then $r(I, \alpha)=-\frac{u_{I I}(I, \alpha)}{u_{I}(I, \alpha)}=\frac{\alpha}{I}>\frac{\beta}{I}=r(I, \beta)$. Let the production function be $y=x L$ where $x=\left\{\begin{array}{ll}0 & \text { with probability } 1-\lambda \\ \bar{x} & \text { with probability } \lambda\end{array}\right.$ and $\lambda \in(0,1)$. We will then show there exists a unique equilibrium wage $w>0$ and $L(w, \alpha) \in\left(0, \frac{A}{w}\right)$ for all $\alpha$.

For each agent $\alpha$, labor demand $L(w, \alpha)$ solves

$$
\operatorname{Max}_{L \in\left[0, \frac{A}{w}\right]} E u=(1-\lambda)(A-w L)^{1-\alpha}+\lambda(A+(\bar{x}-w) L)^{1-\alpha} .
$$

For this concave objective function, the first order condition is sufficient to find $L$ that maximizes $E u$.

$$
\begin{array}{r}
\text { FOC: }\left[\frac{(1-\lambda) w}{\lambda(\bar{x}-w)}\right]^{\frac{1}{\alpha}}(A+(\bar{x}-w) L)=A-w L \\
L(w, \alpha)=\frac{\left(1-k^{\frac{1}{\alpha}}\right) A}{k^{\frac{1}{\alpha}} \bar{x}+\left(1-k^{\frac{1}{\alpha}}\right) w},
\end{array}
$$

where $k \equiv \frac{(1-\lambda) w}{\lambda(\bar{x}-w)}$. If $k \in(0,1)$, then $L(w, \alpha)>0$. Note $k \in(0,1)$ is equivalent to $w \in(0, \lambda \bar{x})$, which implies $\bar{x}>w$ thus making $L(w, \alpha)<\frac{A}{w}$. So $L(w, \alpha) \in\left(0, \frac{A}{w}\right)$ is indeed guaranteed by $w \in(0, \lambda \bar{x})$.

The certainty equivalent wage is defined by

$$
(A+w)^{1-\alpha}=(1-\lambda)[A-w L(w, \alpha)]^{1-\alpha}+\lambda[A+(\bar{x}-w L(w, \alpha))]^{1-\alpha} .
$$

Substituting $L(w, \alpha)$ from (8) yields

$$
(A+w)^{1-\alpha}\left[k^{\frac{1}{\alpha}} \bar{x}+\left(1-k^{\frac{1}{\alpha}}\right) w\right]^{1-\alpha}=(1-\lambda)\left(A k^{\frac{1}{\alpha}} \bar{x}\right)^{1-\alpha}+\lambda(A \bar{x})^{1-\alpha}
$$

If $w=0$, then $k=0$ and $L H S-R H S=-\lambda(A \bar{x})^{1-\alpha}<0$; if $w=\lambda \bar{x}$, then $k=1$ and LHS-RHS= $\left(A \bar{x}+\lambda \bar{x}^{2}\right)^{1-\alpha}-(A \bar{x})^{1-\alpha}>0$. Therefore, by continuity, there must exist a $w \in(0, \lambda \bar{x})$ defined by (9), which guarantees that $L(w, \alpha)$ is an interior solution. 


\section{Conclusion}

We have proved that in the model of $\mathrm{KL}$ an economy-wide increase in risk aversion lowers the wage that clears the labor market, while retaining agent heterogeneity in risk aversion and the endogenous division of agents into less risk averse entrepreneurs and more risk averse workers. This result is of interest in its own right and also appears to fulfill the original vision of KL. By showing that comparative statics are possible in their model without setting aside its fundamental insight, we hope to encourage other researchers to extend their theory, rather than only invoke it as has occurred in the literature to date. We also provided a simple example that establishes the existence of an interior solution to their model and should be useful in the many graduate courses in which KL is taught.

\section{References}

[1] Baron, David P. "Price uncertainty, utility, and industry equilibrium in pure competition." International Economic Review 11 (1970): 463-480.

[2] Evans, David S., and Boyan Jovanovic. "An estimated model of entrepreneurial choice under liquidity constraints." Journal of Political Economy 97 (1989): 808-827.

[3] Kihlstrom, Richard E., and Jean-Jacques Laffont. "A general equilibrium entrepreneurial theory of firm formation based on risk aversion." Journal of Political Economy 87 (1979): 719-748.

[4] Lucas, Robert E. "On the size distribution of business firms." Bell Journal of Economics 9 (1978): 508-523.

[5] Maksimovic, Vojislav, and Gordon Phillips. "Do conglomerate firms allocate resources inefficiently across industries? Theory and evidence." Journal of Finance 57 (2002): 721-767.

[6] Parker, Simon C. The economics of entrepreneurship. Cambridge University Press, 2009. 